\title{
Historische Dynamik und Ambivalenzen der Reform
}

\author{
Ein Kommentar zu den Beiträgen von An- \\ dreas Rieger, Lucien Criblez, Jürgen Kussau \\ und Lutz Oertel zur Entwicklung des \\ Bildungswesens in der Schweiz
}

\section{Peter Drewek}

Formen sich die modernen europäischen Bildungsstrukturen im 19. Jahrhundert im Kontext unterschiedlicher nationaler Konstellationen heraus, scheinen die zunächst verschiedenen Entwicklungsmuster der einzelnen Systeme im Medium von Expansion und Differenzierung, Reform und Integration im 20. Jahrhundert mehr und mehr zu konvergieren. Vor diesem komplexen Hintergrund dekomponieren die vorliegenden Beiträge die Entwicklung des Bildungswesens in der Schweiz unter den Aspekten der langfristigen sozialen Effekte der Bildungsexpansion (Rieger), der Bedeutung von Systemdifferenzierung für die Systemdynamik (Criblez) sowie der Funktionen und Chancen wissenschaftlich fundierter Politikberatung im Reformprozeß (Kussau \& Oertel).

Ausgehend von dem «liberalen Neustart» des Schulwesens im Kanton Zürich 1830 illustriert Rieger die auf einem sehr niedrigen Niveau einsetzende Öffnungsdynamik des Bildungswesens bis in die 1980er-Jahre. Im Ergebnis der statistischen Analysen sind zu diesem Zeitpunkt (fast) alle diejenigen Bildungsbarrieren überwunden, die zuvor im Stadt-Land-Vergleich, zwischen den Konfessionen und zwischen den Geschlechtern Disparitäten der Beteiligung an höherwertigen Bildungskarrieren zur Folge hatten. Mit diesen Indikatoren legt Rieger allerdings ein in den Reformdiskussionen der siebziger Jahre prominentes Modell zugrunde, das für die differenzierte Analyse des langfristigen Abbaus von Bildungsungleichheiten jedoch nicht hinreichend sensibel erscheint. So hat das Bildungssystem für den kollektiven Aufstieg der unteren Sozialschichten, der ländlichen Bevölkerung und lange Zeit auch der Frauen sogar aus den mittleren und höheren Sozialmilieus eine - wie Rieger selbst einführt - nur sehr geringe Bedeutung gehabt. Solange das Bildungswesen aber weder objektiv noch subjektiv als ein realer sozialer Karriereraum gilt, kann die bis weit in das 20. Jahrhundert hinein verschwindend geringe Zahl etwa der Arbeiterkinder in höheren Bildungsgängen nicht als Resultat direkten Ausschlusses interpretiert werden, sondern spiegelt einen historischen Entwicklungsstand, auf dem das Bildungswesen eben nur für Teile der Gesellschaft in seinen kulturell und sozial distinktiven Funktionen überhaupt von Bedeutung war. Sozialgeschichtlich bedeutsamer 
als die geringe Vertretung der Landbevölkerung, der Frauen und der Arbeiterkinder auf den höheren Bildungsstufen scheint vielmehr die sukzessive Durchsetzung des Bildungssystems als eines karriererelevanten Systems überhaupt und die dabei in der Regel überaus konfliktreich verlaufene Integration immer weiterer, nach sozio-ökonomischen Kriterien aber meist bereits bildungsnaher Schichten in die höheren Segmente des Bildungssystems.

In diesem Zusammenhang können die mit vertikal gegliederten Bildungssystemen mehr oder weniger offen verbundenen Selektionsideologien offensichtlich nicht umstandslos mit den faktischen Funktionen solcher Systeme gleichgesetzt werden. Ganz im Gegenteil scheint gerade in der vertikalen Organisation des Schulwesens eine Sogwirkung angelegt, die immer größere Teile der schulpflichtigen Schüler hochschulzugangsorientierte Schullaufbahnen des Sekundarschulwesens besuchen läßt. Rieger demonstriert diesen Prozess indem er die Gesamtquoten bzw. die geschlechtsspezifischen Quoten der Mittelschüler, Diplommittelschüler bzw. Maturitätsmittelschüler als Summe der Einzelquoten der verschiedenen Schultypen berechnet. Noch genauere Ergebnisse wären zu erwarten, wenn sich der Besuch einer bestimmten Schulart auf die jeweils zugrunde liegenden Altersjahrgänge beziehen, d.h. der relative Schulbesuch berechnen liesse.

Die zentrale Frage der (historischen) Bildungsforschung richtet sich im Kontext der Bildungsexpansion seit den sechziger und siebziger Jahren des 20. Jahrhunderts darauf, wieweit sich dabei auch die schichtenspezifischen Chancen zum Erwerb von Hochschulzugangsberechtigungen und Hochschulabschlüssen erhöht haben. So unübersehbar die weiterhin geringfügigen Quoten von Arbeiterkindern an den schweizerischen Mittel- und Maturitätsschulen auch sind, liegen doch auch hier Mobilisierungsprozesse vor, die sich bereits unter Bedingungen einer durchaus selektiv angelegten Struktur vollzogen haben. Für die deutsche Entwicklung ist festgestellt worden, dass die Dynamik des Abbaus sozialer Ungleichheit beim Hochschulzugang gerade in den ersten zweieinhalb Nachkriegsjahrzehnten - unter Bedingungen einer dezidiert selektionsorientierten Schulpolitik und einer vertikal gegliederten Schulstruktur - grösser war als in der Folgeperiode der Bildungsreform der sechziger und siebziger Jahre (Müller, 1998, S. 91). Auch wenn nicht infrage steht, dass schichtspezifische Ungleichheit der Bildungsbeteiligung in hohem Masse fortbesteht, lässt sich die These der durch vertikale Systemstrukturen stabilisierten sozialen Reproduktion offenbar nicht halten. Langfristig dürften zudem aus der Eigendynamik des Bildungswesens weitere Mobilisierungseffekte hervorgehen. Merkmale des Bildungsniveaus der Familie haben in Deutschland für den Schulbesuch der Kinder grösseres Gewicht als Merkmale der beruflichen Stellung (Köhler, 1992, S. 80). Riegers Statistiken belegen, daß auch in der Schweiz schon 1935 die Studentenquoten bei den von höherwertigen Bildungszertifikaten abhängigen Berufen der Techniker, Postbeamten, kaufmännischen Angestellten oder Volksschullehrer mit etwa 10\% zwar noch weit von denen der Ärzte (31\%), Mittelschullehrer (35\%), Ingenieure 
(40\%) oder gar der Hochschullehrer (67\%) entfernt waren, sich aber zugleich bereits überdeutlich von denen der Arbeiter und kleinen Handwerker mit meist nur $1 \%$ abgehoben hatten. Schreibt man den in den sechziger und siebziger Jahren einsetzenden Trend der Bildungsexpansion fort, dürfte das Bildungswesen langfristig an Autonomie gewinnen und seine sozial-reproduktiven Funktionen insofern relativiert werden. Dabei wäre es jedoch verfehlt, das Bildungssystem und seine Strukturen als die einzigen Faktoren sozialer Mobilisierung anzusehen. Gerade in bildungsfernen Schichten hängt die Entscheidung für weiterführende Bildungslaufbahnen einerseits von finanziellen Ressourcen, andererseits von den relativ dazu interpretierten Karriereperspektiven ab. Beide Parameter hängen jedoch in nicht unerheblichem Mass von variierenden Bedingungen ausserhalb des Bildungssystems ab.

Trotz gewisser Ähnlichkeiten in der politischen Legitimation der Bildungsreformen in den sechziger und siebziger Jahren in der Schweiz mit anderen westlichen Industrieländern, insbesondere mit der Bundesrepublik, fallen wenigstens zwei besondere Entwicklungsvoraussetzungen auf. So musste der Neubedarf an akademischen Qualifikationen in der Schweiz in den fünfziger Jahren offenbar nicht zwingend durch einen umgehenden Ausbau der Schul- und Hochschulinstitutionen gedeckt werden, weil die «nötigen Spezialistinnen und Spezialisten [...] problemlos auf dem europäischen Arbeitsmarkt rekrutiert» (Rieger) werden konnten. Dieser Zusammenhang bedürfte insofern weitergehender Untersuchungen, als die damit angesprochene substitutive Funktion von Qualifikationsmärkten anderer Länder das zunächst geringe Reformtempo in der Schweiz über äussere Faktoren mit erklären könnte. Viel weitreichender noch scheinen aber die Hinweise auf den lange Zeit «begrenzten gesellschaftlichen und machtpolitischen Stellenwert der höheren Ausbildung». Wenn «bis in die 50er-Jahre [...] die Universität nur wichtig für den Zugang zu einigen spezialisierten Berufen (Arzt, Jurist, Pfarrer, Ingenieur) sowie zu den Lehrberufen» war und die «S̈̈hne» des «Besitzbürgertums» folglich «überhaupt keine Matura» brauchten, sondern «einen guten Sekundarschulabschluss, einen kaufmännischen Lehrabschluss oder einen Abschluss einer Diplommittelschule, und in der Folge eine individuelle Ausbildung mit Praktika 'von der Pike auf' bei Geschäftsfreunden im In- und Ausland oder im eigenen Betrieb», waren ganz wesentliche Partien des Beschäftigungssystems und der Sozialstruktur vom Bildungssystem und seinen Zertifikaten weitgehend unabhängig. Hatten von den «60-64jährigen Schweizer(n) im obersten Management» nach den Ergebnissen der Volkszählung von 1990 «nur gerade 30\% Hoch- und Fachhochschulabschlüsse» erworben, so galt bis in die Nachkriegzeit hinein eine höhere Schulbildung eben nur «im akademischen Bildungsbürgertum als soziale Norm, jedoch überhaupt nicht beim Besitzbürgertum». Dies erklärt, dass eine «höhere Ausbildung noch keineswegs notwendige Bedingung für einen sozialen Aufstieg» und «vielmehr der Zugang zur Sekundarschule und zu qualitativ hochstehenden Berufslehren und Berufsschulen» «schulpolitisch umkämpft» war. 
Wenn sich seit 1919 zentrale schulpolitische Konflikte - wie Criblez zeigt gleichwohl auf die verschiedenen Typen der Maturitätsschulen bezogen, reflektiert dies den gerade wegen der Segmentierung zwischen bildungs- und besitzbürgerlichen Karriereräumen besonders sensiblen Bereich des Hochschulzugangs als einzigem Aufstiegsweg der nicht zum Besitzbürgertum zählenden Schichten. Dass der mathematisch-naturwissenschaftliche Maturitätstyp C erst 1968 bei der Zulassung zum Medizinstudium anerkannt wurde, demonstriert das bildungspolitisch restriktiv wirksame Gewicht einer einzigen akademischen Profession - der Mediziner - im Reformprozeß, das unter Einbezug des Besitzbürgertums in das höhere Schulwesen und in die Universitäten nicht über einen fünfzigjährigen Zeitraum hinweg so erfolgreich und vor allem so puristisch zu verteidigen gewesen wäre. In Verbindung mit der kurz darauf folgenden Einführung der Maturitätstypen D und E im Jahr 1972 erhöhte sich die Zahl der angebotenen Ausbildungsgänge aller Typen von 132 (1968) auf 391 (1983). Geht man von der Differenz der 259 neu hinzu gekommenen Ausbildungsgänge aus und berechnet ihre Verteilung nach den verschiedenen Typen, entfallen 59\% des Angebotszuwachses auf neu eingerichtete Angebote der Typen C, D und E, dagegen nur 18\% auf den Typ A bzw. 23\% auf den Typ B. Parallel dazu ist die traditionell herausgehobene Stellung der beiden letztgenannten Typen strukturell gestärkt worden. Während sie 1968 75\% aller Ausbildungsgänge der Maturitätsschulen umfaßten, war ihr Anteil 1983 auf 53\% gesunken. Der an seiner Rarität zu messende Angebots "wert» eines Ausbildungsganges verbesserte sich beim Typ A durch die Reduktion von 36\% (48 von 132 angebotenen Ausbildungsgängen im Jahr 1968) auf 24\% (95 von 391 Angeboten 1983).

Expansion durch Differenzierung bedeutet aus dieser Perspektive in erster Linie die Stabilisierung der traditionellen Elitetypen. Es wäre bildungssoziologisch wenig erstaunlich, wenn die soziale Herkunft der Schülerschaft gerade in den traditionellen Maturitätstypen im Verlauf der Expansion zugleich homogener geworden wäre. In zweiter Linie bedeutet Systemdifferenzierung aber nicht nur Kanalisierung, sondern auch Mobilisierung. Wie die Verdoppelung der Zahl der Schülerinnen und Schüler im 10.-13. Schuljahr zwischen 1970 und 1982 erwarten läßt, konnte der von den neuen Maturitätstypen ausgehende Sog für die in der vertikalen Dimension des Schulaufbaus nachgeordneten Schularten nicht folgenlos bleiben. Wie in diesem Zusammenhang gezeigt wird, ging es aber nicht pauschal darum, überhaupt eine mittlere Schulebene zu etablieren. Vielmehr folgt auch dieser Institutionalisierungsprozess einer spezifischen Differenzierungslogik, die Criblez über die Aktivitäten der Gymnasial- bzw. Berufsschullehrer und ihrer Verbände gesteuert sieht. Wenngleich die Etablierung zweier Typen mittlerer Schulen (Diplommittelschule; Berufsmittelschule) durch entsprechend voneinander abgegrenzte Berufsfelder legitimiert und die politischen Interessen der verschiedenen Lehrerkategorien gewahrt werden konnten, führt die über die Fachhochschulen laufende Akademisierung der Anschlusskarrieren letzlich - Criblez bezieht sich auf die Zukunftsperspektiven eines Fach- 
hochschulen und Universitäten weiter annähernden einheitlichen tertiären Bereichs - wieder zu institutioneller Integration.

In der Aufeinanderfolge von Systemdifferenzierung und -integration stellt sich - im deutschen Fall - Differenzierung zunächst als eine Abwehrstrategie der traditionell privilegierten Eliten gegen soziale Aufstiegsgruppen auf der horizontalen Ebene der höheren Bildungseinrichtungen dar, deren mobilisierende Sogwirkungen in der vertikalen Dimension des Bildungssystems durch die nachfolgende Etablierung mittlerer Einrichtungen aufgefangen werden sollten. Ganz entgegen den dahinter stehenden Strategien der Bildungseliten und ihrer politischen Vertreter zur sozialen Kanalisierung aufstiegsorientierter Schichten resultiert der Gesamtprozess aber letztlich in einem generellen upgrading der Bildungsteilnahme.

Ein integrales Moment der Reformprozesse waren nicht nur in der Schweiz Versuche ihrer Rationalisierung durch wissenschaftlich fundierte Bildungsplanung und deren Implementierung in die Bildungspolitik. Wie Kussau und Oertel in differenzierten Analysen zur Programmatik und Entwicklung der Pädagogischen Arbeitsstellen in den Kantonen Bern und Zürich zeigen, erforderte dies von der Bildungspolitik allerdings einen «Spagat zwischen Ausschöpfung der Bildungsreserven und Demokratisierung der Bildungszugänge bei gleichzeitiger Bildungsbegrenzung», der sich auch den Arbeitsstellen als "Widersprüche zwischen demokratisierender und qualifikatorischer Öffnung des Schulsystems» vermittelte. Es ist der Vorzug der sehr gegenstandsnahen Anlage dieses Beitrages, dass in jeder Entwicklungsetappe durch enge Anlehnungen an die Interpretationsansätze der ja überaus breiten Sekundärliteratur die konkreten Entwicklungsprozesse und ihre Analyse unmittelbar aufeinander bezogen werden. Unter operativen Aspekten werden zwar die Ambivalenzen - die "dual-use'-Eigenschaften» - von Informationen in der Bildungspolitik betont, aber trotz aller Skepsis ebenso argumentiert, dass die «funktionale Relevanz von Informationen [...] unumstritten ist» und die "Politik hinter das erreichte Niveau der Informationsverarbeitung nicht mehr zurück» kann. Wenn «das direktdemokratische» im Unterschied zu den «konkurrenzdemokratischen Systemen bis zur jeweiligen Schlussabstimmung keine Mehrheiten» kennt und «Planungsleistungen» deshalb «nur dann Aussicht auf Erfolg» haben, «wenn es ihnen gelingt, im politischen Prozess eine mehrheitsfähige 'Hegemonie' zu gewinnen", so könnte die zukünftig «stärkere Dezentralisierung» im Bildungssystem Richtung und Qualität von Informations- und Entscheidungsprozessen durch die Beteiligung von Lehrern, Eltern und Schülern klientel- und professionsnah reorganisieren.

Die über weite Strecken mit der Abhängigkeit der Bildungsreform von politischen (und ökonomischen) Voraussetzungen argumentierenden Analysen tendieren zugleich zu der Einschätzung, das sich im Zuge von Bildungsreform und -expansion soziale Ungleichheiten im Bildungssystem letztlich eher vergrössert als verkleinert hätten. Abgesehen davon, daß die jüngsten Datensätze in dem als Beleg herangezogenen Zeitschriftenbeitrag aus den frühen achtziger Jahren 
stammen, haben Analysen längerfristiger Entwicklungen die These der persistent inequality in Frage gestellt und dagegen Tendenzen des Abbaus von Bildungsungleichheit betont (Müller, 1998, S. 88 ff.). Angesichts der heute zunehmenden Bedeutung von Bildungspolitik wird nicht zu Unrecht darauf verwiesen, dass es «im Hinblick auf die politische Durchsetzung von Massnahmen zur Ungleichheitsbegrenzung [...] nicht förderlich sein» kann, «der Vorstellung der Unveränderbarkeit der Ungleichheitsrelationen Vorschub zu leisten» (Müller, 1998, S. 90).

\section{Literatur}

Köhler, H. (1992). Bildungsbeteiligung und Sozialstruktur in der Bundesrepublik. Zu Stabilität und Wandel der Ungleichheit von Bildungschancen. Berlin: Max-Planck-Institut für Bildungsforschung.

Müller, W. (1998). Erwartete und unerwartete Folgen der Bildungsexpansion. In J. Friedrichs, M. R. Lepsius \& K. U. Mayer (Hrsg.), Die Diagnosefähigkeit der Soziologie (S. 81-112). Opladen: Westdeutscher Verlag. 